\title{
Fixed width interval estimation for the reciprocal drift of Brownian motion
}

\author{
Robert W. Keener and Michael Woodroofe \\ Department of Statistics, University of Michigan, Ann Arbor, MI 48109, USA
}

Received 1 August 1990; revised manuscript received 19 March 1991

Recommended by T.L. Lai

\begin{abstract}
Let $b(t), 0 \leq t<\infty$, denote Brownian motion with unknown positive drift $\mu$. The problem of setting a fixed width confidence interval for $\theta=1 / \mu$ is considered. The intervals studied are of the form $\left[\hat{\theta}_{\zeta}-h, \hat{\theta}_{\zeta}+h\right]$, where $\zeta$ is a stopping time and $\hat{\theta}_{\zeta}=\zeta / b(\zeta)$. Stopping times $\tau_{h}$ are derived so that these intervals have coverage probabilities converging to a set value $\gamma$ as $h \rightarrow 0$. This convergence is uniform for $\mu$ near 0 . Asymptotic optimality of $\tau_{h}$ is also addressed.
\end{abstract}

AMS Subject Classification: Primary 62L12; secondary 60G40.

Key words: Brownian motion; optimal stopping; sequential estimation; Stein's identity.

\section{Introduction}

Let $b(t), 0 \leq t<\infty$, denote Brownian motion with unknown positive drift $\mu$ and continuous sample paths. Then the expected time to reach a given level $l$ is $l / \mu$, so that estimation of $\theta=1 / \mu$ is of interest.

Natural estimators for $\mu$ and $\theta$ based on $\mathscr{F}_{t}=\sigma(b(s) ; 0<s \leq t)$ are

$$
\hat{\mu}_{t}=\frac{b(t)}{t} \quad \text { and } \quad \hat{\theta}_{t}=\frac{1}{\hat{\mu}_{t}}=\frac{t}{b(t)} .
$$

The problem of setting a fixed width confidence interval for $\theta$ will be considered. Specifically, a stopping time $\zeta$ will be sought such that for all $\mu \in(0, \infty)$,

$$
P_{\mu}\left(\theta \in\left[\hat{\theta}_{\zeta}-h, \hat{\theta}_{\zeta}+h\right]\right) \approx \gamma,
$$

where $h$, the desired half width of the interval, and $\gamma$, the desired coverage probability, are set by the investigator. By Slutsky's theorem,

$$
\sqrt{t}\left(\hat{\theta}_{t}-\theta\right) \Rightarrow \mathrm{N}\left(0, \theta^{4}\right)
$$

as $t \rightarrow \infty$, for any $\mu \in(0, \infty)$. From this approximation, $P\left(\left|\hat{\theta}_{t}-\theta\right| \leq h\right) \approx$ $1-2 \Phi\left(-h \sqrt{t} / \theta^{2}\right)$ which equals $\gamma$ if $c \theta^{2} / \sqrt{t}=h$ where $c=-\Phi^{-1}\left(\frac{1}{2}(1-\gamma)\right)$ and $\Phi$ is 
the standard normal cumulative distribution. This suggests that sampling should be continued until $c \hat{\theta}_{t}^{2} / \sqrt{t} \leq h$. To avoid problems with early stopping and negative estimates, it is convenient to modify this rule to

where

$$
\begin{aligned}
\tau=\tau_{h} & =\inf \left\{t \geq \eta^{4}: \hat{\theta}_{t}>0 \text { and } \frac{c \hat{\theta}_{t}^{2}}{\sqrt{t}} \leq h\right\} \\
& =\inf \left\{t \geq \eta^{4}: b(t) \geq a t^{3 / 4}\right\},
\end{aligned}
$$

and

$$
a=\sqrt{\frac{c}{h}}, \quad \eta=\eta(a) \geq \frac{\log a}{a},
$$

$$
\lim _{a \rightarrow \infty} \frac{\eta}{a}=0 .
$$

In Section 2, the performance of $\tau_{h}$ and the associated confidence interval is studied as $h \downarrow 0$, or equivalently as $a \rightarrow \infty$. Since $\theta \rightarrow \infty$ as $\mu \downarrow 0$, there is concern that approximations for coverage probabilities may break down for $\mu$ near zero. Perhaps surprisingly this is not the case: Theorem 2.1 shows that coverage probabilities for the confidence intervals based on $\tau_{h}$ converge to $\gamma$ uniformly for $\mu$ in any bounded subset of $(0, \infty)$ (actually uniformity holds over sets which grow quickly). In Section 3 , the optimality of $\tau_{h}$ is addressed. If competing stopping times $\zeta_{h}$ have coverage converging to $\gamma$, then $E_{\mu} \zeta_{h}$ cannot be better than $E_{\mu} \tau_{h}$ asymptotically.

The use of sequential methods to produce a uniform limit theorem, where fixed sample size procedures cannot, has been studied by Lai and Siegmund (1983) and Siegmund (1982) in other contexts.

\section{Performance of $\tau$}

Define

$$
\gamma_{h}(\mu)=P_{\mu}\left(\left|\hat{\theta}_{\tau_{h}}-\theta\right| \leq h\right) \quad \text { and } \quad \gamma_{h}^{*}=\inf _{\mu \in(0, \infty)} \gamma_{h}(\mu)
$$

Let

$$
a^{\prime}=\frac{a}{\eta}\left(1-\frac{1}{\sqrt{a \eta}}\right) .
$$

The main result may now be stated.

\section{Theorem 2.1.}

$$
\lim _{h \downarrow 0} \inf \gamma_{h}^{*} \geq \gamma
$$

and

$$
\lim _{h \downarrow 0} \sup _{0<\mu \leq a^{\prime}}\left|\gamma_{h}(\mu)-\gamma\right|=0
$$


Lemma 2.2. For all $1 \leq a<\infty, 0<\mu<\infty$ and $\eta^{4} \leq r<t \leq \infty$,

$$
P_{\mu}\{r<\tau \leq t\} \leq 2\left(1-\Phi\left(a \eta-\mu \eta^{2}\right)\right)+\int_{r}^{t} \frac{a}{s^{3 / 4}} \phi\left(a s^{1 / 4}-\mu \sqrt{s}\right) \mathrm{d} s .
$$

Proof. For fixed $a$ and $\mu$ and for $\eta^{4} \leq r<\infty$, let

Then

$$
\sigma_{r}=\inf \left\{t \geq 0: b(t) \geq a r^{3 / 4}\right\} \text {. }
$$

$$
\{r<\tau \leq t\} \subseteq\left\{\sigma_{\eta^{4}} \leq \eta^{4}\right\} \cup\left\{\sigma_{\eta^{4}}>\eta^{4}, r<\tau \leq t\right\}
$$

Here

$$
\begin{aligned}
P_{\mu}\left\{\sigma_{\eta^{4}} \leq \eta^{4}\right\} & \leq P_{\mu}\left\{\max _{t \leq \eta^{4}} b(t)-\mu t \geq a \eta^{3}-\mu \eta^{4}\right\} \\
& =2\left(1-\Phi\left(a \eta-\mu \eta^{2}\right)\right) .
\end{aligned}
$$

The density for $\sigma_{r}$ under $P_{\mu}$ is well known - see Section 3.2 of Siegmund (1985) for a derivation based on likelihood methods, or Section 7.3 of Karlin and Taylor (1975) for a derivation based on reflection arguments. Integrating this density,

$$
\begin{aligned}
P_{\mu}\left\{\sigma_{\eta^{1}}>\eta^{4}, r<\tau \leq t\right\} & \leq P_{\mu}\left\{r<\sigma_{r} \leq t\right\} \\
& =\int_{r}^{t} \frac{a r^{3 / 4}}{s^{3 / 2}} \phi\left(\frac{a r^{3 / 4}}{\sqrt{s}}-\mu \sqrt{s}\right) \mathrm{d} s .
\end{aligned}
$$

It follows easily that the measure $v$ defined by $v(B)=P_{\mu}\left\{\sigma_{\eta^{4}}>\eta^{4}, \tau \in B\right\}$ for Borel sets $B \subset \mathbb{R}$ is absolutely continuous with respect Lebesgue measure $\lambda$ and that

$$
\frac{\mathrm{d} v}{\mathrm{~d} \lambda}(r)=\lim _{t \downarrow r} \frac{P_{\mu}\left\{\sigma_{\eta^{4}}>\eta^{4}, r<\tau \leq t\right\}}{t-r} \leq \frac{a}{r^{3 / 4}} \phi\left(a r^{1 / 4}-\mu \sqrt{r}\right)
$$

for a.e. $r \in\left(\eta^{4}, \infty\right)$. The lemma follows easily.

Proposition 2.3. For all $1<a<\infty, 0<\mu \leq a / \eta$ and $0<x<\infty$,

$$
P_{\mu}\left\{\tau^{1 / 4}>\frac{a}{\mu}+\frac{x}{a}\right\} \leq 1-\Phi(x) .
$$

Also, for all $0<\mu \leq a^{\prime}$ and $a>\mathrm{e}^{4}$,

$$
P_{\mu}\left\{\tau^{1 / 4} \leq \frac{a}{\mu}-\frac{1}{\sqrt{\mu}}\right\} \leq 11(1-\Phi(\sqrt{a \eta}))+4 a \phi(\sqrt{a}-1)
$$

Proof. For the first assertion, let

$$
r^{1 / 4}=\frac{a}{\mu}+\frac{x}{a} \geq \eta .
$$

Then

$$
P_{\mu}\left\{\tau^{1 / 4}>\frac{a}{\mu}+\frac{x}{a}\right\}=P_{\mu}\{\tau>r\}
$$




$$
\begin{aligned}
& \leq P_{\mu}\left\{b(r)<a r^{3 / 4}\right\} \\
& =1-\Phi\left(\mu \sqrt{r}-a r^{1 / 4}\right) .
\end{aligned}
$$

The last expression is at most $1-\Phi(x)$ since

$$
\mu \sqrt{r}-a r^{1 / 4}=x+\mu \frac{x^{2}}{a^{2}} \geq x .
$$

For the second assertion, assume $\mathrm{e}^{4} \leq a<\infty$ and $0<\mu \leq a^{\prime}$ and let

Then

$$
t^{1 / 4}=\frac{a}{\mu}-\frac{1}{\sqrt{\mu}} .
$$

$$
t^{1 / 4}=\frac{a}{\mu}\left(1-\frac{\sqrt{\mu}}{a}\right) \geq \frac{a}{\mu}\left(1-\frac{1}{\sqrt{a \eta}}\right) \geq \eta .
$$

By Lemma 2.2 with $r=\eta^{4}$ and first principles,

$$
\begin{aligned}
P_{\mu}\left\{\tau^{1 / 4} \leq \frac{a}{\mu}-\frac{1}{\sqrt{\mu}}\right\} & \leq P_{\mu}\left\{\eta^{4}<\tau \leq t\right\}+P_{\mu}\left\{\tau=\eta^{4}\right\} \\
& \leq 3\left(1-\Phi\left(a \eta-\mu \eta^{2}\right)\right)+\int_{\eta^{4}}^{t} \frac{a}{s^{3 / 4}} \phi\left(a s^{1 / 4}-\mu \sqrt{s}\right) \mathrm{d} s .
\end{aligned}
$$

The first term here is easy to bound: since $\mu \leq a^{\prime}$,

$$
1-\Phi\left(a \eta-\mu \eta^{2}\right) \leq 1-\Phi\left(a \eta-a^{\prime} \eta^{2}\right)=1-\Phi(\sqrt{a \bar{\eta}}) .
$$

The argument $g(s)=a s^{1 / 4}-\mu \sqrt{s}$ of $\phi$ in the integral in (2.3) is a quadratic function of $s^{1 / 4}$ which achieves its maximum at $s_{0}=(a /(2 \mu))^{4}$ and decreases for $s \geq s_{0}$. Since

$$
g(t)=\frac{a}{\sqrt{\mu}}-1 \geq \sqrt{a \eta}-1 \geq 0
$$

and since $\phi$ is decreasing on $(0, \infty), \phi(g(s)) \leq \phi(g(t))$ for $s \in\left(s_{0}, t\right)$. Therefore

$$
\begin{aligned}
\int_{s_{0}}^{t} \frac{a}{s^{3 / 4}} \phi\left(a s^{1 / 4}-\mu \sqrt{s}\right) \mathrm{d} s & \leq \phi(g(t)) \int_{0}^{t} \frac{a}{s^{3 / 4}} \mathrm{~d} s \\
& =4 a t^{1 / 4} \phi\left(\frac{a}{\sqrt{\mu}}-1\right) .
\end{aligned}
$$

To simplify this bound, note that $x^{2} \phi(x-1)$ has derivative $-x(x+1)(x-2) \phi(x-1)$ which is negative for $x>2$. Since $a>\mathrm{e}^{4}$ gives $\sqrt{a \eta}>2$,

$$
\sup _{x \geq \sqrt{a \eta}} x^{2} \phi(x-1)=\operatorname{a\eta } \phi(\sqrt{a \eta}-1) \text {. }
$$

Consequently, since $\mu \leq a^{\prime}$ implies $\mu<a / \eta$ and $a / \sqrt{\mu}>\sqrt{a \eta}$,

$$
\int_{s_{0}}^{t} \frac{a}{s^{3 / 4}} \phi\left(a s^{1 / 4}-\mu \sqrt{s}\right) \mathrm{d} s \leq 4 a \frac{a}{\mu} \phi\left(\frac{a}{\sqrt{\mu}}-1\right)
$$




$$
\begin{aligned}
& \leq 4 \sup _{x \geq \sqrt{a \eta}} x^{2} \phi(x-1) \\
& =4 a \eta \phi(\sqrt{a \eta}-1) .
\end{aligned}
$$

To bound the integral in (2.3) over $s \in\left(\eta^{4}, s_{0}\right)$, note that for $s \leq s_{0}, g(s)=$ $a s^{1 / 4}\left(1-\mu s^{1 / 4} / a\right) \geq a s^{1 / 4}\left(1-\mu s_{0}^{1 / 4} / a\right)=a s^{1 / 4} / 2 \geq 0$. Hence

$$
\begin{aligned}
\int_{\eta^{4}}^{s_{0}} \frac{a}{S^{3 / 4}} \phi\left(a s^{1 / 4}-\mu \sqrt{s}\right) \mathrm{d} s & \leq \int_{\eta^{4}}^{s_{0}} \frac{a}{s^{3 / 4}} \phi\left(\frac{1}{2} a s^{1 / 4}\right) \mathrm{d} s \\
& =4 \int_{a \eta}^{a s_{1}^{1 / 4}} \phi\left(\frac{1}{2} x\right) \mathrm{d} x \\
& \leq 8\left(1-\Phi\left(\frac{1}{2} a \eta\right)\right) \\
& \leq 8(1-\Phi(\sqrt{a \eta})),
\end{aligned}
$$

the last inequality since $\sqrt{a \eta}>2$. Proposition 2.3 now follows using (2.4), (2.5) and (2.6) in (2.3).

Define

$$
\tau^{*}=\frac{\mu^{4} \tau}{a^{4}}
$$

and let

$$
b^{*}(t)=-\frac{\mu^{2}}{a^{2}}\left[b\left(\frac{a^{4} t}{\mu^{4}}\right)-\frac{a^{4} t}{\mu^{3}}\right]
$$

for $0 \leq t<\infty$. Note that $\left\{b^{*}(t)\right\}_{t \geq 0}$ is a standard Brownian motion under $P_{\mu}$ for any $a>0$ and any $\mu \in(0, \infty)$.

Corollary 2.4. For any $\varepsilon>0$,

Also,

$$
\lim _{a \rightarrow \infty} \sup _{0<\mu \leq a^{\prime}} P_{\mu}\left\{\left|\tau^{*}-1\right| \geq \varepsilon\right\}=0 .
$$

$$
\lim _{a \rightarrow \infty} \sup _{0<\mu \leq a^{\prime}} E_{\mu}\left|\tau^{*}-1\right|=0
$$

Proof. The first assertion follows easily from Proposition 2.3. Then for the second assertion, uniform integrability for the variables $\left(\tau^{*}-1\right)^{+}$is sufficient, and this also follows easily from Proposition 2.3.

Proposition 2.5. For any $\varepsilon>0$,

$$
\lim _{a \rightarrow \infty} \sup _{0<\mu \leq a^{\prime}} P_{\mu}\left\{\left|a^{2}\left(\hat{\theta}_{\tau}-\theta\right)-b^{*}(1)\right| \geq \varepsilon\right\}=0 .
$$

Proof. Observe that on $\left\{\tau>\eta^{4}\right\}$, 
and

$$
\hat{\theta}_{\tau}-\theta=\frac{\tau}{b(\tau)}-\frac{1}{\mu}=\frac{\mu \tau-b(\tau)}{\mu b(\tau)}=\frac{\mu \tau-b(\tau)}{\mu a \tau^{3 / 4}}
$$

$$
\begin{aligned}
a^{2}\left(\hat{\theta}_{\tau}-\theta\right) & =-\left(\frac{a^{3}}{\mu^{3} \tau^{3 / 4}}\right) \times\left(\frac{\mu^{2}}{a^{2}}\right)[b(\tau)-\mu \tau] \\
& =\left(\frac{1}{\tau^{*}}\right)^{3 / 4} b^{*}\left(\tau^{*}\right),
\end{aligned}
$$

so

$$
a^{2}\left(\hat{\theta}_{\tau}-\theta\right)-b^{*}(1)=\left(\frac{1}{\tau^{*}}\right)^{3 / 4}\left[b^{*}\left(\tau^{*}\right)-b^{*}(1)\right]+\left[\left(\frac{1}{\tau^{*}}\right)^{3 / 4}-1\right] b^{*}(1) .
$$

Assume now that $\delta<\frac{1}{2}$. For $x \in\left[\frac{1}{2}, \frac{3}{2}\right]$ the function $x^{-3 / 4}$ has derivative between -3 and 0 , so $\left\{\left|\tau^{*}-1\right|<\delta\right\}$ implies $\left|\tau^{*^{-3 / 4}}-1\right|<3 \delta$. Hence on $\left\{\left|\tau^{*}-1\right|<\delta, \tau>\eta^{4}\right\}$,

$$
\left|a^{2}\left(\hat{\theta}_{\tau}-\theta\right)-b^{*}(1)\right| \leq\left(\frac{1}{1-\delta}\right)^{3 / 4} \sup _{|t-1|<\delta}\left|b^{*}(t)-b^{*}(1)\right|+3 \delta\left|b^{*}(1)\right|,
$$

and therefore (taking advantage of the fact that probability statements concerning $b^{*}$ are independent of $\mu$ )

$$
\begin{aligned}
& \sup _{0<\mu \leq a^{\prime}} P_{\mu}\left\{\left|a^{2}\left(\hat{\theta}_{\tau}-\theta\right)-b^{*}(1)\right| \geq \varepsilon\right\} \\
& \leq \sup _{0<\mu \leq a^{\prime}} P_{\mu}\left\{\left|\tau^{*}-1\right| \geq \delta\right\}+\sup _{0<\mu \leq a^{\prime}} P_{\mu}\left\{\tau=\eta^{4}\right\}+P\left\{3 \delta\left|b^{*}(1)\right|>\frac{1}{2} \varepsilon\right\} \\
& \quad+P\left\{\left(\frac{1}{1-\delta}\right)^{3 / 4} \sup _{|t-1|<\delta}\left|b^{*}(t)-b^{*}(1)\right|>\frac{1}{2} \varepsilon\right\} .
\end{aligned}
$$

The proposition follows using Corollary 2.4, letting $a \rightarrow \infty$ and then $\delta \downarrow 0$.

Proof of Theorem 2.1. Since $a^{2} h=c$,

$$
\gamma_{h}(\mu)=P_{\mu}\left\{\left|a^{2}\left(\hat{\theta}_{\tau}-\theta\right)\right| \leq c\right\}
$$

and (2.2) follows directly from Proposition 2.5 because $b^{*}(1)$ has the standard normal distribution. So it suffices to show (2.1) with $\inf _{0<\mu<\infty}$ replaced by inf $a_{a^{\prime}<\mu<\infty}$. The proof is similar to that of Proposition 2.5. Let

and

$$
\tau_{*}=\frac{\tau}{\eta^{4}}
$$

$$
b_{*}(t)=\frac{b\left(\eta^{4} t\right)-\mu \eta^{4} t}{\eta^{2}}
$$

for $0 \leq t<\infty$, so $b_{*}$ is standard Brownian motion for any $\mu \in(0, \infty)$. In this case,

$$
a^{2}\left|\hat{\theta}_{\tau}-\theta\right| \leq a^{2}\left|\frac{\mu \tau-b(\tau)}{a \mu \tau^{3 / 4}}\right|=\frac{a\left|b_{*}\left(\tau_{*}\right)\right|}{\mu \eta \tau_{*}^{3 / 4}} \leq \frac{\left|h_{*}\left(\tau_{*}\right)\right|}{\tau_{*}^{3 / 4}(1-1 / \sqrt{a \eta})}
$$


for all $\mu \in\left(a^{\prime}, \infty\right)$. So it suffices to show that $\tau_{*} \rightarrow 1$ in $P_{\mu}$-probability, uniformly for $\mu \in\left(a^{\prime}, \infty\right)$. This follows because $\tau_{*} \geq 1$ and if $\mu \geq a^{\prime}$ and $\varepsilon>0$,

$$
\begin{aligned}
P_{\mu}\left\{\tau_{*}^{1 / 4} \geq 1+\varepsilon\right\} & \leq P_{a^{\prime}}\left\{b\left(\eta^{4}(1+\varepsilon)^{4}\right) \leq a \eta^{3}(1+\varepsilon)^{3}\right\} \\
& =\Phi\left\{a \eta(1+\varepsilon)-a^{\prime} \eta^{2}(1+\varepsilon)^{2}\right\}
\end{aligned}
$$

which is independent of $\mu$ and approaches zero as $a \rightarrow \infty$.

\section{Optimality of $\tau$}

In this section the asymptotic optimality of $\tau$ will be studied as $h \rightarrow 0$. The main result, Theorem 3.1, shows that $\tau$ is asymptotically optimal for a class of stopping problems under a wide class of prior distributions. From this, if $\zeta_{h}$ are competing stopping times for which

$$
\liminf _{h \downarrow 0} P_{\mu}\left\{\left|\hat{\theta}_{\zeta_{h}}-\theta\right| \leq h\right\} \geq \gamma
$$

for all $\mu \in(0, \infty)$, then

$$
\liminf _{h \downarrow 0} \sup _{\mu \in C} \frac{E_{\mu} \zeta_{h}}{E_{\mu} \tau_{h}} \geq 1,
$$

for any nondegenerate, compact subinterval $C \subset(0, \infty)$ (no matter how small).

In the sequel, $P$ will denote probability under a Bayesian model in which $\mu$ is a random variable with prior density $\xi$. The key to showing optimality of $\tau$ is to find a stopping problem for which $\tau$ is the solution. This idea is developed for fixed width interval estimation of a normal mean in Woodroofe (1986). Here such a stopping problem may be constructed by letting the loss for stopping at time $t$ be

$$
L_{h}(t)=K I\left\{\left|\hat{\theta}_{t}-\theta\right| \geq h\right\}+t h^{2} \mu^{4},
$$

where $K=c / \phi(c)$. The risk of a stopping time $\zeta$ is then

$$
\bar{r}_{h}(\zeta)=E L_{h}(\zeta)
$$

Theorem 3.1. Suppose $\xi$ is absolutely continuous on $\mathbb{R}$ with support a subset of $\left[\mu_{0}, \mu_{1}\right], 0<\mu_{0}<\mu_{1}<\infty$ and that $\xi$ has finite Fisher information, i.e., $E\left(\xi^{\prime}(\mu) / \xi(\mu)\right)^{2}<$ $\infty$. Then

$$
\lim _{h \downarrow 0} \inf _{\zeta} \bar{r}_{h}(\zeta)=\lim _{h \downarrow 0} \bar{r}_{h}\left(\tau_{h}\right)=K(1-\gamma)+c^{2}
$$

To see why the stated optimality for $\tau$ follows from this theorem, suppose (3.2) fails for a nondegenerate, compact subinterval $C_{0} \subset(0, \infty)$ and stopping times $\zeta_{h}$ satisfying (3.1). Using Corollary 2.4, there exists $\varepsilon>0$ and a positive sequence $h_{n} \downarrow 0$ as $n \rightarrow \infty$ such that

$$
E_{u} \zeta_{h_{n}} \leq(1-\varepsilon) a^{4} / u^{4}
$$


for all $n \geq 1$ and all $u \in C_{0}$. Let $\xi_{0}$ be a density which satisfies the conditions of Theorem 3.1 and vanishes off $C_{0}$. With this density,

$$
\begin{aligned}
\limsup _{n \rightarrow \infty} \bar{r}_{h_{n}}\left(\zeta_{h_{n}}\right) & =\limsup _{n \rightarrow \infty} \int_{C_{0}}\left[K P_{u}\left\{\left|\hat{\theta}_{\zeta_{n}}-\theta\right| \geq h_{n}\right\}+\frac{c^{2} u^{4}}{a^{4}} E_{u} \zeta_{h_{n}}\right] \xi_{0}(u) \mathrm{d} u \\
& \leq K(1-\gamma)+(1-\varepsilon) c^{2}
\end{aligned}
$$

by dominated convergence, which contradicts Theorem 3.1 .

The second equality in Theorem 3.1 will follow from the results of the previous section. So to establish the theorem an asymptotic lower bound for the risk of an arbitrary stopping time is needed. The general idea is to approximate the posterior distribution of $\theta$ at a time $t$ by $\mathrm{N}\left(\hat{\theta}_{t}, \hat{\theta}_{t}^{4} / t\right)$. When $\zeta$ is large, this leads to the approximation

where

$$
\begin{aligned}
E\left[L_{h}(\zeta) \mid \mathscr{F}_{\zeta}\right] & \approx 2 K \Phi\left(-h \sqrt{\zeta} / \hat{\theta}_{\zeta}^{2}\right)+\zeta h^{2} / \hat{\theta}_{\zeta}^{4} \\
& =H\left(h \sqrt{\zeta} / \hat{\theta}_{\zeta}^{2}\right),
\end{aligned}
$$

Since

$$
H(x)=2 K \Phi(-x)+x^{2}
$$

$$
H^{\prime}(c)=-2 K \phi(c)+2 c=0
$$

and for $x>0$,

$$
H^{\prime \prime}(x)=2 K x \phi(x)+2>0
$$

we have

$$
\inf _{x \geq 0} H(x)=H(c)=K(1-\gamma)+c^{2}
$$

Hence the main issue proving Theorem 3.1 is justifying the approximation (3.3). A key tool is the integration by parts formula in the following proposition which extends Lemma 1 of Stein (1986). For a proof see Section 4 of Woodroofe (1990). Our notation identifies measures $v$ with the corresponding linear operator in the usual way: $v f=\int f \mathrm{~d} v$. Let $\mathscr{H}_{p}$ denote the class of all measurable functions $g$ such that $\sup _{x \in(\infty, \infty)} g(x) /\left(1+|x|^{p}\right)<\infty$, and let $\mathscr{H}=\bigcup_{p \geq 0} \mathscr{H}_{p}$.

Proposition 3.2 (Stein's identity). Suppose $\mathrm{d} \Gamma=f \mathrm{~d} \Phi, f$ is absolutely continuous on $\mathbb{R}$ and

$$
\int\left|f^{\prime}(x) x^{p}\right| \mathrm{d} \Phi(x)<\infty
$$

for some integer $p \geq 0$. Then

$$
\Gamma g=\Gamma 1 \times \Phi g+\int U g \times \frac{f^{\prime}}{f} \mathrm{~d} \Gamma
$$

for all $g \in \mathscr{H}_{p+1}$, where

$$
U g(x)=\int_{x}^{\infty}(g(y)-\Phi g) \phi(y) \mathrm{d} y / \phi(x) .
$$


The linear operator $U$ that appears in this lemma has a number of nice properties. By Lemma 3 of Stein (1986),

$$
\sup _{x \in \mathbb{R}}|U g(x)| \leq \sqrt{2 \pi} \sup _{x \in \mathbb{R}}|g(x)| \text {. }
$$

Also for integral $p \geq 2$, integration by parts gives

and hence

$$
\int_{x}^{\infty} y^{p} \phi(y) \mathrm{d} y / \phi(x)=x^{p-1}+(p-1) \int_{x}^{\infty} y^{p-2} \phi(y) \mathrm{d} y / \phi(x),
$$

$$
U x^{p}=x^{p-1}+(p-1) U x^{p-2}
$$

Hence $U$ maps polynomials into polynomials. Since $U x=1$, this recursion gives $U x^{2}=x, U x^{3}=x^{2}+2$ and $U x^{4}=x^{3}+3 x$.

The following corollary shows how Stein's identity can be used to study posterior distributions. Similar results appear as Proposition 4 of Woodroofe (1990) and Proposition 1 of Woodroofe and Hardwick (1990). The linear operator $U$ will now act on functions of three arguments and we will write $U f(z, x, y)$ as a shorthand for $U f(\cdot, x, y)(z)$. Let $Z_{t}=\sqrt{t}\left(\mu-\hat{\mu}_{t}\right)$.

Corollary 3.3. Suppose $E\left|g\left(Z_{t}, \hat{\mu}_{t}, t\right)\right|<\infty$ and $g(\cdot, x, t) \in \mathscr{H}$ for a.e. $x \in \mathbb{R}$. If $\xi$ satisfies the conditions in Theorem 3.1 then

$$
E\left[g\left(Z_{t}, \hat{\mu}_{t}, t\right) \mid \mathscr{F}_{t}\right]=\Phi g\left(\cdot, \hat{\mu}_{t}, t\right)+\frac{1}{\sqrt{t}} E\left[U g\left(Z_{t}, \hat{\mu}_{t}, t\right) \frac{\xi^{\prime}(\mu)}{\xi(\mu)} \mid \mathscr{F}_{t}\right] .
$$

Proof. Let $f_{t}(z)=f_{t}(z, \hat{\mu})$ denote the conditional density of $Z_{t}$ given $\mathscr{F}_{t}$ with respect to $\Phi$. Then $f_{t}(z)$ is proportional to $\xi\left(\hat{\mu}_{t}+z / \sqrt{t}\right)$ and hence

$$
\frac{f_{t}^{\prime}(z)}{f_{l}(z)}=\frac{1}{\sqrt{t}} \frac{\xi^{\prime}\left(\hat{\mu}_{t}+z / \sqrt{t}\right)}{\xi\left(\hat{\mu}_{t}+z / \sqrt{t}\right)} \text {. }
$$

By Stein's identity,

$$
\begin{aligned}
E\left[g\left(Z_{t}, \hat{\mu}_{t}, t\right) \mid \mathscr{F}_{t}\right] & =\int g\left(z, \hat{\mu}_{t}, t\right) f_{t}(z) \mathrm{d} \Phi(z) \\
& =\Phi g\left(\cdot, \hat{\mu}_{t}, t\right)+\frac{1}{\sqrt{t}} \int U g\left(z, \hat{\mu}_{t}, t\right) \frac{\xi^{\prime}\left(\hat{\mu}_{t}+z / \sqrt{t}\right)}{\xi\left(\hat{\mu}_{t}+z / \sqrt{t}\right)} f_{t}(z) \mathrm{d} \Phi(z) \\
& =\Phi g\left(\cdot, \hat{\mu}_{t}, t\right)+\frac{1}{\sqrt{t}} E\left[U g\left(Z_{t}, \hat{\mu}_{t}, t\right) \frac{\xi^{\prime}(\mu)}{\xi(\mu)} \mid \mathscr{F}_{t}\right] .
\end{aligned}
$$

By the Schwarz inequality,

$$
\int\left|\xi^{\prime}(x)\right| \mathrm{d} x \leq\left\{\int \xi(x) \mathrm{d} x\right\}^{1 / 2}\left\{\int \frac{\xi^{\prime}(x)^{2}}{\xi(x)} \mathrm{d} x\right\}^{1 / 2},
$$

so the regularity condition for Stein's identity holds because $f_{t}^{\prime}(z) \propto \xi^{\prime}\left(\hat{\mu}_{t}+z / \sqrt{t}\right)$ and for any $p \geq 0$, 


$$
\int\left|\xi^{\prime}\left(\hat{\mu}_{t}+x / \sqrt{t}\right) x^{p}\right| \mathrm{d} \Phi(x) \leq\left\{\sup _{x \in(-\infty, \infty)}|x|^{p} \phi(x)\right\} \times \sqrt{t} \int\left|\xi^{\prime}(x)\right| \mathrm{d} x<\infty .
$$

\section{Proof of Theorem 3.1. Since}

$$
\bar{r}_{h}\left(\tau_{h}\right)=\int\left[K\left(1-\gamma_{h}(u)\right)+c^{2} E_{u} \tau^{*}\right] \xi(u) \mathrm{d} u,
$$

the second equality in the theorem follows immediately from Theorem 2.1 and Corollary 2.4. To verify the other equality, let $\zeta=\zeta_{h}$ be stopping times indexed by $h$. Since these stopping times are arbitrary, it is sufficient to show that

$$
\liminf _{h \backslash 0} \bar{r}_{h}(\zeta) \geq K(1-\gamma)+c^{2}
$$

The prcvious corollary to Stein's identity gives a useful approximation for conditional risks for a stopping time $\zeta$ when $\zeta$ is large. When $\zeta$ is small a separate argument is needed. Let

$$
\xi_{t}(x)=\frac{\xi(x) \mathrm{e}^{b(t) x-t x^{2} / 2}}{\int \xi(y) \mathrm{e}^{b(t) y-t y^{2} / 2} \mathrm{~d} y},
$$

a conditional density of $\mu$ given $\mathscr{F}_{t}$, and let

If $\bar{\xi}=\sup \xi$, then

$$
\Lambda_{T}=\sup _{i \leq T} \sup _{x \in\left[\mu_{0}, \mu_{1}\right]} \xi_{t}(x)
$$

$$
\begin{aligned}
\sup _{x \in\left[\mu_{0}, \mu_{1}\right]} \xi_{t}(x) & \leq \bar{\xi} \frac{\sup _{x \in\left[\mu_{0}, \mu_{1}\right]} \mathrm{e}^{b(t) x-t x^{2} / 2}}{\inf _{x \in\left[\mu_{0}, \mu_{1}\right]} \mathrm{e}^{b(t) x-t x^{2} / 2}} \\
& \leq \bar{\xi} \exp \left\{t\left(\mu_{1}^{2}-\mu_{0}^{2}\right)+|b(t)|\left(\mu_{1}-\mu_{0}\right)\right\},
\end{aligned}
$$

and hence $\Lambda_{T}<\infty$ almost surely, since $b(t)$ is continuous in $t$. Since the conditional density for $\theta$ given $\mathscr{F}_{t}$ is at most $\xi_{t} / \mu_{0}^{2}$, conditioning on $\mathscr{F}_{\zeta}$,

$$
P\left[\left|\hat{\theta}_{\zeta}-\theta\right| \leq h, \zeta \leq T\right] \leq E \min \left\{1, h \Lambda_{T} / \mu_{0}^{2}\right\} \rightarrow 0
$$

as $h \downarrow 0$. By a diagonalization argument we can find $1 \leq T_{h} \rightarrow \infty$ such that

$$
\sup _{\zeta} P\left[\left|\hat{\theta}_{\zeta}-\theta\right| \leq h, \zeta \leq T_{h}\right] \rightarrow 0
$$

as $h \downarrow 0$, which implies

as $h \downarrow 0$.

$$
P\left[\left|\hat{\theta}_{\zeta}-\theta\right|>h, \zeta \leq T_{h}\right]-P\left(\zeta \leq T_{h}\right) \rightarrow 0
$$

Next, define functions $g_{0}$ and $g_{1}$ by

and

$$
g_{0}\left(z_{t}, \hat{\mu}_{t}, t\right)=I\left\{\left|\frac{1}{\hat{\mu}_{t}}-\frac{1}{\hat{\mu}_{t}+Z_{t} / \sqrt{t}}\right|>h\right\}=I\left\{\left|\hat{\theta}_{t}-\theta\right|>h\right\}
$$

$$
g_{1}\left(Z_{t}, \hat{\mu}_{t}, t\right)=t\left(\hat{\mu}_{t}+Z_{t} / \sqrt{t}\right)^{4}=t \mu^{4} \text {. }
$$


Then

$$
\begin{aligned}
\bar{r}_{h}(\zeta) \geq & K P\left[\left|\hat{\theta}_{\zeta}-\theta\right|>h, \zeta \leq T_{h}\right]+K E\left[g_{0}\left(Z_{\zeta}, \hat{\mu}_{\zeta}, \zeta\right) ; \zeta>T_{h}\right] \\
& +h^{2} E\left[g_{1}\left(Z_{\zeta}, \hat{\mu}_{\zeta}, \zeta\right) ; \zeta>T_{h}\right]
\end{aligned}
$$

By Corollary 3.3, for $i=0$ or 1 ,

$$
\begin{aligned}
& E\left[g_{i}\left(Z_{\zeta}, \hat{\mu}_{\zeta}, \zeta\right) ; \zeta>T_{h}\right] \\
& \quad=E\left[\Phi g_{i}\left(\cdot, \hat{\mu}_{\zeta}, \zeta\right) ; \zeta>T_{h}\right]+E\left[\frac{1}{\sqrt{\zeta}} U g_{i}\left(Z_{\zeta}, \hat{\mu}_{\zeta}, \zeta\right) \frac{\xi^{\prime}(\mu)}{\xi(\mu)} ; \zeta>T_{h}\right] .
\end{aligned}
$$

By (3.5), $\left|g_{0}\right| \leq 1$ implies $\left|U g_{0}\right|<\sqrt{2 \pi}$ and therefore

$$
\left|E\left\{\frac{1}{\sqrt{\zeta}} U g_{0}\left(Z_{\zeta}, \hat{\mu}_{\zeta}, \zeta\right) \frac{\xi^{\prime}(\mu)}{\xi(\mu)} ; \zeta>T_{h}\right\}\right| \leq \frac{\sqrt{2 \pi}}{\sqrt{T_{h}}} E\left|\frac{\xi^{\prime}(\mu)}{\xi(\mu)}\right| \rightarrow 0
$$

as $h \downarrow 0$. Hence

$$
K E\left[g_{0}\left(Z_{\zeta}, \hat{\mu}_{\zeta}, \zeta\right) ; \zeta>T_{h}\right]-K E\left[\Phi g_{0}\left(\cdot, \hat{\mu}_{\zeta}, \zeta\right) ; \zeta>T_{h}\right] \rightarrow 0
$$

as $h \downarrow 0$. On $\left\{0<\hat{\mu}_{t}<1 / h\right\}$,

$$
\Phi g_{0}\left(\cdot, \hat{\mu}_{\zeta}, \zeta\right)=\Phi\left(-\sqrt{\zeta} \frac{h \hat{\mu}_{\zeta}^{2}}{1-h \hat{\mu}_{\zeta}}\right)+\Phi\left(-\sqrt{\zeta} \frac{h \hat{\mu}_{\zeta}^{2}}{1+h \hat{\mu}_{\zeta}}\right) .
$$

Now $\Phi(x)-\Phi(y) \rightarrow 0$, uniformly in $x$ and $y$ as $x / y \rightarrow 1$. Consequently

$$
\begin{aligned}
& {\left[\Phi\left(-\sqrt{\zeta} \frac{h \hat{\mu}_{\zeta}^{2}}{1-h \hat{\mu}_{\zeta}}\right)+\Phi\left(-\sqrt{\zeta} \frac{h \hat{\mu}_{\zeta}^{2}}{1+h \hat{\mu}_{\zeta}}\right)-2 \Phi\left(-\sqrt{\zeta} h \hat{\mu}_{\zeta}^{2}\right)\right]} \\
& \quad \times I\left\{\hat{\mu}_{\zeta} \in\left[\frac{1}{2} \mu_{0}, 2 \mu_{1}\right]\right\} \rightarrow 0 .
\end{aligned}
$$

By the strong law of large numbers, $P\left(\hat{\mu}_{\zeta} \notin\left[\frac{1}{2} \mu_{0}, 2 \mu_{1}\right], \zeta>T_{h}\right) \rightarrow 0$ as $h \downarrow 0$, so

$$
K E\left[g_{0}\left(Z_{\zeta}, \hat{\mu}_{\zeta}, \zeta\right) ; \zeta>T_{h}\right]-2 K E\left[\Phi\left(-\sqrt{\zeta} h \hat{\mu}_{\zeta}^{2}\right) ; \zeta>T_{h}\right] \rightarrow 0
$$

as $h \downarrow 0$. From the formulae for $U x^{p}$,

$$
U g_{1}\left(Z_{\zeta}, \hat{\mu}_{\zeta}, \zeta\right)=\sqrt{\zeta} p_{1}\left(\mu, \hat{\mu}_{\zeta}\right)+p_{2}\left(\mu, \hat{\mu}_{\zeta}\right) / \sqrt{\zeta},
$$

where $p_{1}\left(\mu, \hat{\mu}_{\zeta}\right)=\hat{\mu}_{\zeta}^{3}+\hat{\mu}_{\zeta}^{2} \mu+\hat{\mu}_{\zeta} \mu^{2}+\mu^{3}$ and $p_{2}\left(\mu, \hat{\mu}_{\zeta}\right)=3 \mu+5 \hat{\mu}_{\zeta}$. Since $\xi$ has compact support and since $\sup _{t \geq 1}\left|\hat{\mu}_{t}-\mu\right|$ has moments of all orders, $\sup h E\left[p^{2}\left(\mu, \hat{\mu}_{\zeta}\right)\right]<\infty$ for any polynomial $p$. Hence

$$
\begin{aligned}
h^{2} & \left|E\left\{\frac{1}{\sqrt{\zeta}} U g_{1}\left(Z_{\zeta}, \hat{\mu}_{\zeta}, \zeta\right) \frac{\xi^{\prime}(\mu)}{\xi(\mu)} ; \zeta>T_{h}\right\}\right| \\
& \leq h^{2} E\left[\left(\left|p_{1}\left(\mu, \hat{\mu}_{\zeta}\right)\right|+\left|p_{2}\left(\mu, \hat{\mu}_{\zeta}\right)\right|\right)\left|\frac{\xi^{\prime}(\mu)}{\xi(\mu)}\right| ; \zeta>T_{h}\right] \\
& \leq h^{2} \sqrt{2 E\left[p_{1}^{2}\left(\mu, \hat{\mu}_{\zeta}\right)+p_{2}^{2}\left(\mu, \hat{\mu}_{\zeta}\right) ; \zeta>T_{h}\right] E\left(\xi^{\prime}(\mu) / \xi(\mu)\right)^{2}} \\
& \rightarrow 0
\end{aligned}
$$


as $h \downarrow 0$. Consequently

$$
h^{2} E\left[g_{1}\left(Z_{\zeta}, \hat{\mu}_{\zeta}, \zeta\right) ; \zeta>T_{h}\right]-E\left[\Phi g_{1}\left(\cdot, \hat{\mu}_{\zeta}, \zeta\right) ; \zeta>T_{h}\right] \rightarrow 0
$$

as $h \downarrow 0$. Direct calculation gives

so

$$
\Phi g_{1}\left(\cdot, \hat{\mu}_{\zeta}, \zeta\right)=\zeta \hat{\mu}_{\zeta}^{4}+6 \hat{\mu}_{\zeta}^{2}+3 / \zeta
$$

and

$$
h^{2} E\left[\Phi g_{1}\left(\cdot, \hat{\mu}_{\zeta}, \zeta\right) ; \zeta>T_{h}\right]-h^{2} E\left[\zeta \hat{\mu}_{\zeta}^{4} ; \zeta>T_{h}\right] \rightarrow 0
$$

$$
h^{2} E\left[g_{1}\left(Z_{\zeta}, \hat{\mu}_{\zeta}, \zeta\right) ; \zeta>T_{h}\right]-h^{2} E\left[\zeta \mu_{\zeta}^{4} ; \zeta>T_{h}\right] \rightarrow 0
$$

as $h \downarrow 0$. Using this cquation, (3.6) and (3.8) in (3.7), and using (3.4),

$$
\begin{aligned}
\liminf _{h \downarrow 0} \bar{r}_{h}(\zeta) & \geq \liminf _{h \downarrow 0}\left\{K P\left(\zeta \leq T_{h}\right)+E\left[2 K \Phi\left(\sqrt{\zeta} h \hat{\mu}_{\zeta}^{2}\right)+\zeta h^{2} \hat{\mu}_{\zeta}^{4} ; \zeta>T_{h}\right]\right\} \\
& \geq \liminf _{h \downarrow 0}\left\{K P\left(\zeta \leq T_{h}\right)+\left(K(1-\gamma)+c^{2}\right) P\left(\zeta>T_{h}\right)\right\} \\
& \geq K(1-\gamma)+c^{2} .
\end{aligned}
$$

The last inequality follows from (3.4) since $K=H(0) \geq H(c)=K(1-\gamma)+c^{2}$. This proves the theorem.

\section{References}

Karlin, S. and H. Taylor (1975). A First Course in Stochastic Processes. Academic Press, New York. Lai, T.L. and D. Siegmund (1983). Fixed accuracy estimation of an autoregressive parameter. Ann. Statist. 11, 478-485.

Siegmund, D. (1982). A sequential confidence interval for the odds ratio. Probab. Math. Siatist. 2, 149-156.

Siegmund, D. (1985). Sequential Analysis: Tests and Confidence Intervals. Springer, New York.

Stein, C. (1986). Approximate Computation of Expectations. IMS, Hayward, CA.

Woodroofe, M. (1986). Asymptotic optimality in sequential interval estimation. Adv. Appl. Math. 7 , $70-79$.

Woodroofe, M. (1990). Integrable expansions for posterior distributions for one parameter exponential families. Statist. Sinica. To appear.

Woodroofe, M. and J. Hardwick (1990). Sequential allocation for an estimation problem with ethical costs. Ann. Statist. 18, 1358-1377. 